\title{
An Adult's Vitiligo in Estonia: Study of 155 Patients
}

\author{
M. Karelson ${ }^{*}, 1$, K. Kingo ${ }^{1,2}$, T. Salum ${ }^{3}$, S. Kõks ${ }^{2,4,5}$ and H. Silm ${ }^{1}$ \\ ${ }^{I}$ Department of Dermatology and Venerology, University of Tartu, Estonia \\ ${ }^{2}$ Centre of Molecular and Clinical Medicine, University of Tartu, Estonia \\ ${ }^{3}$ Tartu University Hospital, United Laboratories, Estonia \\ ${ }^{4}$ Department of Physiology, University of Tartu, Estonia \\ ${ }^{5}$ Institute of Veterinary Medicine and Animal Sciences, Estonia, University of Life Sciences, Estonia
}

\begin{abstract}
Background: Vitiligo is a common depigmentary disorder characterized by white patches of the skin, hair and mucous membranes due to selective destruction of melanocytes.

Objective: The objective of this study was to analyze the clinical characteristics, coexisting diseases, presence of autoantibodies and autoimmune polyglandular syndrome (APS) in Estonian adult vitiligo patients.

Methods:Adult patients with vitiligo were called to participate in the study at the Dermatology Department of Tartu University from January 2005 to July 2008. One hundred fifty five subjects were examined in 141 of those the level of thyroid peroxidase antibodies (TPO-Ab), gastric parietal cell antibodies (PCA), antinuclear antibodies (ANA), antiadrenal cortex antibodies (AAA) and rheumatoid factor (RF) in blood were measured.

Results: Study group (mean age 44.9 years, mean age of vitiligo onset 28.5 years, mean duration of vitiligo 16.9 years) consisted of 44 males and 111 females. Vitiligo vulgaris was the most common clinical type (81.3\%), followed by acrofacial, focal, segmental and universal vitiligo. Two-thirds of subjects reported a coexisting disease and $36.7 \%$ had one or more disease of autoimmune origin. The presence of autoantibodies was established in 49.6\%. TPO-Ab was found in $36.9 \%$, PCA in $14.2 \%$, ANA and AAA both in $2.8 \%$ and positive RF in $7.8 \%$ cases. 17 subjects had APS 3,35 had subclinical APS 3 and two subjects had APS 4.
\end{abstract}

Conclusions: Vitiligo vulgaris was the most frequent clinical type. Vitiligo was associated with other autoimmune diseases, the presence of autoantibodies in the blood was frequent (especially TPO-Ab) and many subjects had APS.

Keywords: Vitiligo, clinical types, coexisting disease, autoantibodies, autoimmune polyglandular syndrome.

\section{INTRODUCTION}

Vitiligo is a common acquired depigmentary disorder characterized by white patches of the skin, hair and mucous membranes due to selective destruction of melanocytes. It affects $0.5-1 \%$ of the worldwide population and ranges up $8.8 \%$ in India $[1,2]$. Recent publications from China have shown that these numbers could be overestimated as they have found the prevalence of vitiligo to be $0.09-0.19 \%[3,4]$

In most cases disease appears before the age of 20 but it can start after fifty [5, 6]. There is still no uniform understanding of vitiligo clinical subtypes. Koga divides vitiligo into nonsegmental (A) and segmental (B) types based on the different distribution pattern and clinical course [7]. Hann and Nordlund created more complicated classification of vitiligo. According to the pattern of distribution they differentiated disease as localized, generalized and universal. Localized vitiligo can further

*Address correspondence to this author at the Department of Dermatology and Venerology, University of Tartu, 31 Raja Street, 50417 Tartu, Estonia; Tel: +3 727319 710; Fax: +3 727319701 ;

E-mail: maire.karelson@kliinikum.ee divide into focal (one or a few macules in nondermatomal distribution), segmental (one or more macules in dermatomal distribution), mucosal (depigmentation of lips, oral mucosa and genitals) and generalized vitiligo divides into acrofacial (distal parts of the extremities and face), vulgaris (scattered macules all over the body) and mixed (different types at the same time) types [8]. In universal type nearly complete or complete depigmentation of the skin appears. The clinical phenotype of vitiligo can be changed during the years [9, $10]$.

Based on autoimmune hypothesis there are several studies focused on associated diseases in vitiligo patients and their families. Alkhateeb has found the elevation of several autoimmune disorders: vitiligo itself, thyroiditis, pernicious anemia, Addison's disease, systemic lupus erythematosus and inflammatory bowel disease [11]. Other investigators have added to this list diabetes, rheumatoid arthritis, alopecia areata, psoriasis, chronic urticaria, asthma [12-14]. Results of the studies from different geographical regions are still controversial and needs clarification. Vitiligo is a part of autoimmune polyglandular syndromes (APS), most frequently of APS-3 (coexisting with autoimmune thyroid diseases) $[15,16]$. 
The objective of this study was to present the clinical characteristics, coexisting diseases, presence of autoantibodies and APS in Estonian adult vitiligo patients.

\section{MATERIALS AND METHODOLOGY}

Estonia is a small Baltic state with a population 1.4 million people. Adult patients with vitiligo were called to participate in the study all over the country. The data were collected from January 2005 to July 2008. The study was approved by the Ethical Review Committee on Human Research of the University of Tartu and all participants signed a written informed consent.

Patients were examined at the Dermatology Department of Tartu University by an experienced dermatologist. The questionnaire, including age, sex, nationality, skin phototype, site of onset of vitiligo, duration, concomitant diseases, familial history, spontaneous repigmentation, previous treatment, was completed by a dermatologist. The diagnosis of vitiligo was based on characteristic loss of skin pigmentation with typical localization and the examination under Wood's lamp. The clinical types of vitiligo were classified as focal (one or few macules in a nondermatomal distribution), segmental (unilateral segmental distribution), acrofacial (distal extremities and face), vulgaris (scattered over the body), universal (over 90\% depigmentation). The presence of leukotrichia, Koebner phenomenon, and halo nevi was noted. The evolution of vitiligo considered active when new lesions appeared and existing lesions increased in size over the past 3 months. Vitiligo was stable when depigmentation did not increase during the last 3 months.

Antinuclear antibodies (ANA), gastric parietal cell antibodies (PCA) and anti-adrenal cortex antibodies (AAA) were determined by indirect immunofluorescence method using rat liver as antigenic substrate for ANA and mouse stomach as antigenic substrate for detection of PCA. AAA was detected on normal human adrenal tissue [17, 18]. Polyclonal rabbit anti-human IgG conjugated to fluorescein isothiocyanate (DAKO, Glostrup, Denmark) was used as secondary antibody for detection of all these antibodies. CLIA, Immulite 2000 (Siemens Medical Solutions Diagnostics) was used for the detection of thyroid peroxidase antibodies (TPO-Ab) and immunoturbidimetric assay, Cobas Integra 400 Plus (Roche Diagnostics $\mathrm{GmbH}$ ) for the assessment of rheumatoid factor (RF) [19].

\section{RESULTS}

In total, 155 patients, 44 males (28.4\%) and 111 females (71.6\%), were enrolled. The mean age of patients was 44.9 years (age range $18-82$ years) and the mean age of vitiligo onset was 28.5 years (age range 2-76 years). The mean ages of onset for males and for females were 28.2 and 28.6, respectively. The mean duration of vitiligo was 16.9 years (0.5 -58 years) for all patients, 16.2 years for males and 17.2 years for females. 51 subjects $(32.9 \%)$ had skin phototype II, 102 subjects $(65.8 \%)$ had skin phototype III and 2 subjects $(1.3 \%)$ had skin phototype IV.

Vitiligo vulgaris was the most common clinical type, observed in 126 cases ( $81.3 \%$ ), followed by acrofacial, focal, segmental and universal vitiligo. Patients with segmental and universal vitiligo were affected earlier than other types of vitiligo (Table 1).
Table 1. Comparisons of Mean Ages of Onset of Different Types of Vitiligo

\begin{tabular}{|c|c|c|}
\hline Clinical Types Vitiligo & Number (\%) & Mean Age (y) of Onset \\
\hline \hline Vulgaris & $126(81.3)$ & 28.0 \\
Universal & $5(3.2)$ & 14.6 \\
Acrofacial & $12(7.7)$ & 44.2 \\
Segmental & $5(3.2)$ & 7.6 \\
Focal & $7(4.5)$ & 34.7 \\
Total & $155(100)$ & 28.5 \\
\hline
\end{tabular}

The most common site of onset vitiligo was the upper limb in 55 patients (35.5\%), followed by trunk in $37(23.9 \%)$, lower limb in $27(17.4 \%)$, head and neck in $23(14.8 \%)$, body folds in $13(8.4 \%)$ patients. Twenty patients $(12.9 \%)$ showed Koebner's phenomenon. Disease was active and progressed in $109(70.3 \%)$ subjects, who confirmed the appearance of new lesions or increase in the size of existing lesions within the past 3 months.

At the time of the study, vitiligo initial clinical type had been transformed in 23 cases (14.8\%). Vitiligo vulgaris was the initial clinical type of 113 subjects $(72.9 \%)$; five of them developed universal vitiligo. 12 cases of focal vitiligo and 6 cases of acrofacial vitiligo transformed to vitiligo vulgaris. Segmental vitiligo did not transform to other subtype.

According to the extent of depigmentation, patients with minimal disease predominated. In 82 cases $(52.9 \%)$ up to $10 \%$ body surface area was involved. Hair depigmentation in vitiligo lesions were seen in 75 cases $(48.4 \%)$ and mucosal involvement in 14 cases $(9.0 \%)$.

60 subjects $(38.7 \%)$ named factors that had brought on their vitiligo initially or worsened it. These were mechanical injury in 15 cases, psychological trauma in 15 cases, hormonal changes in 13 cases, sunburn in 6 cases, UV radiation and radiation in 6 cases, skin irritation in 3 cases, drug intake in 3 cases, and other disease in one case. 54 patients $(34.8 \%)$ had got some kind of treatment but without good effect. 43 subjects $(27.7 \%)$ had noticed partial unstable spontaneous repigmentation in some of their lesions during the course of vitiligo.

In all, 104 patients $(67.1 \%)$ self-reported a coexisting disease and 57 of those (36.7\%) had one ore more disease of autoimmune origin. Halo nevi were seen in 24 cases (15.5\%). 26 subjects $(16.8 \%)$ gave a personal history of thyroid disease, 20 of them had been diagnosed autoimmune thyroiditis. Other coexisting diseases were hypertonia in 24 cases, allergic conditions in 36 cases, psoriasis in 7 cases, rheumatoid arthritis and diabetes both in 6 cases, oncological and hematological diseases in 8 cases, alopecia areata, ancylosing spondylitis and pernicious anemia all in 2 cases (Table 2).

The same diseases could be found in their first and second degree relatives. 40 patients $(26.0 \%)$ gave a family history of vitiligo, $32(20.8 \%)$ of thyroid disease, $21(13.6 \%)$ of diabetes, $13(8.4 \%)$ of psoriasis, $7(4.5 \%)$ of rheumatoid arthritis, 53 (34.4\%) of hypertonia (Table 2). One subject did not know her relatives. 
Table 2. Vitiligo Associated Diseases

\begin{tabular}{|c|c|c|}
\hline Associated Diseases & Number of Vitiligo Subjects & No of Vitiligo Subjects whose Relatives has the Disease \\
\hline Halo nevus & 24 & 2 \\
\hline Thyroid disease & 28 & 31 \\
\hline Diabetes & 6 & 21 \\
\hline Rheumatoid arthritis & 6 & 7 \\
\hline Psoriasis & 7 & 13 \\
\hline Gastroduodenal ulcer & 2 & 0 \\
\hline Pernicious anemia & 2 & 0 \\
\hline Alopecia areata & 2 & 2 \\
\hline Ankylosing spondylitis & 2 & 0 \\
\hline Multiplex sclerosis & 1 & 0 \\
\hline Sclerodermia & 1 & 1 \\
\hline Neurofibromatosis & 1 & 0 \\
\hline Addison disease & 0 & 1 \\
\hline Urticaria & 8 & 0 \\
\hline Fast type allergy (hair, dust, pollen) & 7 & 1 \\
\hline Asthma & 5 & 3 \\
\hline Atopic dermatitis & 2 & 0 \\
\hline Drug allergy & 14 & 0 \\
\hline Oncological and hematological disease & 8 & 3 \\
\hline Hypertonia & 24 & 53 \\
\hline
\end{tabular}

The levels of TPO-Ab, PCA, ANA, AAA and RF in blood were measured of 141 subjects. The presence of autoantibodies was established in 70 cases. TPO-Ab were found in 52 subjects, PCA in 20 subjects, ANA and AAA both in 4 subjects and 11 had positive for RF. Concurring positivity of TPO-Ab and PCA was found in 12 cases; TPO$\mathrm{Ab}$ and AAA in two cases, TPO-Ab and RF in one case. Blood of one subject was positive for four measured antibodies (Table 3).

Table 3. The Presence of Autoantibodies in the Blood

\begin{tabular}{|l|c|}
\hline \multicolumn{1}{|c|}{ Autoantibodies Type } & No. of Subjects (\%) \\
\hline \hline TPO-Ab & $52(36.9)$ \\
PCA & $20(14.2)$ \\
AAA & $4(2.8)$ \\
ANA & $4(2.8)$ \\
RF & $11(7.8)$ \\
TPO-Ab and PCA & $12(8.5)$ \\
TPO-Ab and AAA & $2(1.4)$ \\
TPO-Ab and RF & $1(0.7)$ \\
TPO-Ab, PCA, AAA, ANA & $1(0.7)$ \\
\hline
\end{tabular}

According to the results of autoantibodies and the list of coexisting disorders we found that 17 subjects had APS 3, 35 had subclinical APS 3 and two subjects had APS 4 (Table 4).

\section{DISCUSSION}

Vitiligo affects both sexes equally, but some studies have shown the female preponderance $[20,21]$. Our study group consisted of II and III skin phototype European Caucasians where females dominated $(2.5: 1)$ and the reason of this superiority can be the concern of cosmetic looks by the women. This extra-organized study for the adults showed older mean age of the subjects (44.9 years) and long-lasting disease (up to 58 years) comparing to the other studies [2, $22]$. Our data confirmed that the peak incidence of vitiligo is in the second or third decade of life $[23,24]$. In $38.7 \%$ of cases vitiligo appeared at the age of 20 and in $58.7 \%$ of cases at the age of 30 . In $14.8 \%$ of cases vitiligo started after fifties and this was higher then $6.8 \%$ reported by Dogra [6]. The mean age of onset was same for the males and females.

Vitiligo vulgaris was the most common clinical type, observed in $81.3 \%$ of cases, followed by acrofacial, focal, segmental and universal vitiligo in descending order by frequency. Vitiligo vulgaris has been the most frequent clinical pattern in many published papers comprising in 39$83 \%$ of subjects with vitiligo $[2,6,9,25]$. We did not see local mucosal type and mixed vitiligo in our subjects. Segmental vitiligo was rare in our adult group and has been previously reported $0-4.5 \%$ in adults $[6,25]$. We did not see segmental pattern in the face region, shown by Hann as the most frequent localisation in Korean patients [26]. The initial site of vitiligo was the upper limbs in one-third of the subjects, followed by the trunk, lower limbs, head and neck, body folds. Previous studies have found vitiligo initial sites to be limbs and face $[2,27,28]$. All these localizations are trauma - prone sites. Koebner's sign stayed between 5-30\% described by the others $[29,30]$. The number of subjects with leucotrichia was high, similar to Indian patients shown by Dogra and Dave [6, 31]. We got an opposite results of mucosal involvement. Only $9 \%$ of subjects had additional mucosal depigmentation. Others have observed mucosal involvement between 7.4 - 74\% [24, 29].

Vitiligo was active in $70.3 \%$ of the subjects, similar results have published by Dave and Arycan [27, 31]. In spite of the long mean duration of vitiligo (16.9 years), subjects 
Table 4. APS Types and Subtypes in Vitiligo

\begin{tabular}{|c|c|c|}
\hline Type of APS & Associated Disease & No. of Subjects \\
\hline \hline APS-3C & Autoimmune thyroiditis & 13 \\
APS-3C, subclinical & Positive TPO-Ab & 35 \\
APS-3 C + A & Autoimmune thyroiditis, multiplex sclerosis, diabetes & 1 \\
APS-3 D + C & Autoimmune thyroiditis, rheumatoid arthritis & 3 \\
APS- 4 & Alopecia areata & 2 \\
\hline
\end{tabular}

with minimal extension predominated. More than half of cases had up to $10 \%$ body surface area involvement; additional $32.9 \%$ had skin involvement between $11 \%$ and $50 \%$. Approximately three- quarters of the subjects reported that vitiligo had started as a vulgar clinical type and acquired symmetrical distribution during one-two years. Handa has shown vitiligo vulgaris approximately $70 \%$ of the subjects with average disease duration only 3.7 years [2]. Contrary to Liu, who found the initial clinical type to be focal vitiligo in three - quarters in his study group, only in $12.3 \%$ of cases focal vitiligo was reported as onset type of vitiligo in our subjects [9]. Several studies have shown earliest onset of segmental vitiligo and we agree with this [26, 32]. Subjects with segmental and universal vitiligo were affected earlier than other types of vitiligo. Universal vitiligo showed a longlasting course with an average 30.8 years, but mean durability of focal vitiligo was only 4.6 years. It confirms the opinion that the disease has a slowly progressive and chronic long course.

Two-thirds of the subjects reported coexisting diseases and every third patient had diagnosed at least one disease of autoimmune origin, including halo nevi. We saw halo nevi in $15.5 \%$ of cases, mostly one-two but in two patients numerously. In 9 cases of vitiligo, including these two, halo nevi were the first depigmented lesions on the skin before vitiligo appeared. Studies have shown the presence of halo nevi in $1-35 \%$ of vitiligo patients but seldom in adults [22, 33]. Autoimmune thyroiditis has been the most frequent disease, associated up to $34 \%$ of vitiligo patients in different studies around the world, except in China [13, 25, 34]. In our study $16.8 \%$ of the subjects gave a personal history of thyroid disease and $12.9 \%$ were aware of autoimmune thyroiditis, having APS-3C. Studies from India and Nigeria showed lower $(0.5-0.6 \%)$ incidence of thyroid disease because the screening was done only patients with clinical features $[2,25]$. The number of subjects having vitiligo in their relatives was quite high $(26.0 \%)$ as the positive family incidences stay between $10-36 \%[22,35]$. The prevalence of thyroid disease $(20.1 \%)$, diabetes $(13.5 \%)$, psoriasis $(8.4 \%)$ and rheumatoid arthritis $(4.5 \%)$ among the relatives in our study was high too, as already shown by the others [12-14].

The presence of measured autoantibodies was established in $49.6 \%$ of our adult group. The level of TPO-Ab in the blood was raised in 52 subjects, 35 of them were without clinical signs of thyroid disease and had not been checked before. As the TPO-Ab is the hallmark of autoimmune thyroiditis, we can assume that these 35 subjects had subclinical autoimmune thyroid disease and they belong to type APS-3C. According to the new APS classification by Betterle we discovered that one subject had APS-3 $\mathrm{C}+\mathrm{A}$, three had APS-3 D+C and two had APS-4 [15, 16]. Subjects with positive AAA and TPO-Ab needed further consultation suspected as having APS-2 (Addison disease and autoimmune thyroiditis). The elevation of rheumatoid factor was mild in 10 cases, except one subject with very high value and undiagnosed rheumatoid arthritis.

\section{CONCLUSION}

Our study affirmed several well-known and some new aspects of vitiligo. Vitiligo vulgaris was the most frequent clinical type. Disease had long-lasting course with a slow progression during the years as absolute majority showed pigment loss less then $10 \%$ of body area. Vitiligo was associated with other autoimmune diseases, the presence of autoantibodies in the blood was frequent (especially TPOAb) and many subjects had APS.

\section{ACKNOWLEDGEMENTS}

This study was financially supported by the target based funding from the Estonian Ministry of Education grant No SF0180043s07, by the Estonian Science Foundation Grant No 6576 and by the Centre of Molecular and Clinical Medicine Grant VARMC-TIPP.

\section{REFERENCES}

[1] Lerner AB, Nordlund JJ. Vitiligo. What is it? Is it important? JAMA 1978; 239: 1183-7.

[2] Handa S, Kaur I. Vitiligo: clinical findings in 1436 patients. J Am Acad Dermatol 1999; 26: 653-7.

[3] Lu T, Gao T, Wang A, et al. Vitiligo prevalence study in Shaanxi Province, China. Int J Dermatol 2007; 46: 47-51.

[4] Xu YY, Ye DQ, Tong ZC, et al. An epidemiological survey on four skin diseases in Anhui. Chin J Dermatol 2002; 35: 406-7.

[5] Jaisankar TJ, Baruah MC, Garg BR. Vitiligo in children. Int J Dermatol 1992; 31: 612-23.

[6] Dogra S, Parsad D, Handa S, et al. Late onset vitiligo: A study of 182 patients. Int J Dermatol 2005; 44: 193-6.

[7] Koga M. Vitiligo: a new classification and therapy. Br J Dermatol 1977; 97: 255-61.

[8] Hann SK, Nordlund JJ. Clinical features of generalized vitiligo. In: Hann SK, Nordlund JJ, Eds. Vitiligo. UK, Oxford: Blackwell Science Ltd 2000; 35-48.

[9] Liu JB, Li M, Yang S, et al. Clinical profiles of vitiligo in China: an analysis of 3742 patients. Clin and Exp Dermatol 2005; 30: 32731 .

[10] Zaima H, Koga M. Clinical course of 44 cases of localized type vitiligo. J Dermatol 2002; 29: 15-9.

[11] Alkhateeb A, Fain PR, Thody A, et al. Epidemiology of vitiligo and associated autoimmune diseases in Caucasians probands and their families. Pigment Cell Res 2003; 16: 208-14.

[12] Gould IM. Vitiligo in diabetes mellitus. Br J Dermatol 1985; 113 : 153-5.

[13] Cunliffe WJ, Hall R, Newell DJ, et al. Vitiligo, thyroid disease and autoimmunity. Br J Dermatol 1968; 80: 135-9.

[14] Zhang Z, Xu SX, Zhang FY, et al. The analysis of genetics and associated autoimmune diseases in Chinese vitiligo patients. Arch Dermatol Res 2009; 30: 167-73.

[15] Betterle C, Dal PC, Mantero F, et al. Autoimmune adrenal insufficiency and autoimmune polyendocrine syndromes: 
autoantibodies, autoantigens, and their applicability in diagnosis and disease prediction. Endocr Rev 2002; 23: 237-64.

[16] Amerio P, Tracanna M, Remigis P De, et al. Vitiligo associated with other autoimmune diseases: polyglandular autoimmune syndrome types 3B+C and 4. Clin Exp Dermatol 2006; 31: 746-9.

[17] Uibo R, Talja I, Jõgi R, et al. Autoantibodies in Estonia and Sweden, Population with Different Responses to Allergens. Int Arch Allergy Immunol 1998; 117: 126-30.

[18] Betterle C, Zanchetta R, Chen S, Furmaniak J. Antibodies to adrenal, gonadal tissues and steroidogenic enzymes. In: Shoenfeld Y, Gershwin ME, Meroni PL, Eds. Autoantibodies. USA: Elsevier 2007; 389-401.

[19] Laulu SL, Slev PR, Roberts WL. Perfomance characteristics of 5 automated thyroglobulin autoantibody and thyroid peroxidase autoantibody assays. Clin Chim Acta 2007; 376: 88-95.

[20] Kovacs SO. Vitiligo. J Am Acad Dermatol 1998; 38: 647-6.

[21] Jaigirdar MQ, Alam SM, Maidul AZ. Clinical presentation of vitiligo. Mymensingh Med J 2002; 11: 79-81.

[22] Martis J, Bhat R, Nandakishore B, et al. A clinical study of vitiligo. Indian J Dermatol Venereol Leprol 2002; 68: 92-3.

[23] Bleehen SS, Anstey AV. Disorders of Skin Colour. In: Burns T, Breathnack S, Cox N, Griffiths C, Eds. Rookś Textbook of Dermatology. USA: Blackwell Science Ltd 2004; 39: pp. 53-6.

[24] Onunu AN, Kubeyinje EP. Vitiligo in Nigerian African: a study of 351 patients in Benin City, Nigeria. Int J Dermatol 2003; 42: 800-2.

[25] Mason CP, Gawkrodger DJ. Vitiligo presentation in adults. Clin Exp Dermatol 2005; 3: 344-5.
[26] Hann SK, Lee HJ. Segmental vitiligo: Clinical findings in 208 patients. J Am Acad Dermatol 1996; 35: 671-4.

[27] Arycan O, Koc K, Ersoy L. Clinical characteristics in 113 Turkish vitiligo patients. Acta Dermatovenerol Alp Panonica Adriat 2008; 17: 129-32.

[28] Akrem J, Baroudi A, Aichi T, Houch F, Hamdaoui MH. Profile of vitiligo in the South Tunesia. Int J Dermatol 2008; 47: 670-4.

[29] Hann SK, Chun WH, Park YK. Clinical characteristics of progressive vitiligo. Int J Dermatol 1997; 36: 353-5.

[30] Taieb A, Picardo M. The definition and assessment of vitiligo: a consensus report of the Vitiligo European Task Force. Pigment Cell Res 2007; 20: 27-35.

[31] Dave S, Thappa DM, Souza DM. Clinical predictors of otcome invitiligo. Indian J Dermatol Venereol Leprol 2002; 68: 323-5.

[32] Barona MI, Arrunategui A, Falabella R, et al. An epidemiologic case-control study in a population with vitiligo. J Am Acad Dermatol 1995; 33: 621-5.

[33] Sehgal VN, Srivastava G. Vitiligo: Compendium of clinicoepidemiological features. Indian J Dermatol Venereol Leprol 2007; 73: $149-55$.

[34] Schallreuter KU, Lemke R, Brandt O, et al. Vitiligo and other diseases: coexistence or true association? Hamburg study on 321 patients. Dermatology 1994; 188: 269-75.

[35] Gopal KV, Rama Rao GR, Kumar JH, et al. Vitiligo: A part of a systemic autoiummune process. Indian J Dermatol Venereol Leprol 2007; 73: 162-5.

(C) Karelson et al.; Licensee Bentham Open.

This is an open access article licensed under the terms of the Creative Commons Attribution Non-Commercial License (http://creativecommons.org/licenses/by$\mathrm{nc} / 3.0 /$ ) which permits unrestricted, non-commercial use, distribution and reproduction in any medium, provided the work is properly cited. 